\title{
Deletion diagnostics in fitting a given regression model to a new observation
}

\author{
Myung Geun Kim ${ }^{1, a}$ \\ ${ }^{a}$ Department of Mathematics Education, Seowon University, Korea
}

\begin{abstract}
A graphical diagnostic method based on multiple case deletions in a regression context is introduced by using the sampling distribution of the difference between two least squares estimators with and without multiple cases. Principal components analysis plays a key role in deriving this diagnostic method. Multiple case deletions of test statistic are also considered when a new observation is fitted to a given regression model. The result is useful for detecting influential observations in econometric data analysis, for example in checking whether the consumption pattern at a later time is the same as the one found before or not, as well as for investigating the influence of cases in the usual regression model. An illustrative example is given.
\end{abstract}

Keywords: case deletions, covariance matrix, influence, principal components analysis, test statistic

\section{Introduction}

Regression analysis has been used widely in econometric field. We often have a question, "Is an economic relationship at a later time the same as the one found before?". When we use a linear regression to represent an economic relationship, a statistical expression of this question is to say "Does an observation at a later time follow the regression model estimated by the data obtained before?". This question can be answered statistically in a regression context by taking two steps. The first step is to establish a regression model among economic factors of interest and the second step is to check whether an observation at hand follows this established regression model, by using an appropriate test of hypotheses given in for example Chow (1960) or Ghilagaber (2004). Specific examples are, "Is the consumption pattern at a later time the same as the one found before?", "Is the dependency of the price of a commodity at present on some economic factors the same as the one found before?", and so on.

In a regression context, it is well known that one observation or a few observations can substantially influence the least squares estimators and their relevant quantities (Chatterjee and Hadi, 1988; Cook and Weisberg, 1982). Hence in an analysis of regression data, it is very important to detect such observations and to assess their influence on diverse regression quantities. In our problem, there may exist some influential observations in each of two steps mentioned above. Even a single influential observation in either step can lead us to a wrong analysis result. The first step of our analysis is just the usual regression analysis for which a diagnostic method based on multiple case deletions is introduced in Section 3 by using the sampling distribution of the difference between two least squares

\footnotetext{
${ }^{1}$ Department of Mathematics Education, Seowon University, 377-3 Musimseoro, Heungdeok-gu, Cheongju 28674, Korea.

E-mail: mgkim@seowon.ac.kr
}

Published 31 May 2016 / journal homepage: http://csam.or.kr

(c) 2016 The Korean Statistical Society, and Korean International Statistical Society. All rights reserved. 
estimators with and without multiple cases. Principal components analysis plays a key role in deriving this diagnostic method. As in the usual regression analysis, some observations can have a large influence in testing whether an observation at hand comes from a given regression model. Hence a suitable method of detecting influential observations is needed for the second step. To this end multiple case deletions of test statistic are derived in Section 4. In Section 5, a numerical example is provided for illustration.

\section{Preliminaries}

We consider the multiple linear regression model defined by

$$
y=X \beta+\varepsilon,
$$

where $y=\left(y_{1}, \ldots, y_{n}\right)^{T}$ is a column vector of response variables of size $n, X=\left(x_{1}, \ldots x_{n}\right)^{T}$ is an $n \times p$ full column rank matrix of $n$ measurements on $p$ fixed regressors, $\beta$ is a column vector of $p$ unknown regression coefficients of size $p$, and $\varepsilon=\left(\varepsilon_{1}, \ldots, \varepsilon_{n}\right)^{T}$ is a column vector of unobservable random errors of size $n$. The errors $\varepsilon_{1}, \ldots, \varepsilon_{n}$ are assumed to be independent and identically distributed as a normal distribution $N\left(0, \sigma^{2}\right)$ with zero mean and variance $\sigma^{2}$.

We denote the least squares estimator of $\beta$ by $\hat{\beta}=\left(X^{T} X\right)^{-1} X^{T} y$. The residual vector is given by $e=\left(e_{1}, \ldots, e_{n}\right)^{n}=y-X \hat{\beta}=\left(I_{n}-H\right) y$, where $H=\left(h_{i j}\right)=X\left(X^{T} X\right)^{-1} X^{T}$ is the projection matrix and $I_{n}$ is the identity matrix of order $n$. We have an alternative expression $e_{i}=y_{i}-x_{i}^{T} \hat{\beta}$. The residual sum of squares is written as $s^{2}=(y-X \hat{\beta})^{T}(y-X \hat{\beta})=e^{T} e$. An unbiased estimator of $\sigma^{2}$ is $\hat{\sigma}^{2}=s^{2} /(n-p)$. The covariance matrix of $\hat{\beta}$ becomes $\operatorname{cov}(\hat{\beta})=\sigma^{2}\left(X^{T} X\right)^{-1}$. More details can be found in Seber (1977).

\section{Influence in estimating regression coefficients}

Let $J$ be an index set of $k$ indices among $1, \ldots, n$. We denote by $y_{J}$ the column vector formed by the elements of $y$ corresponding to $J$ and let $X_{J}$ consist of the rows of $X$ indexed by $J$. When we write as $y_{(J)}$ the vector $y$ from which $y_{J}$ are removed and as $X_{(J)}$ the matrix $X$ from which the rows of $X_{J}$ are removed, we have

$$
X^{T} X=X_{(J)}^{T} X_{(J)}+X_{J}^{T} X_{J}
$$

so that

$$
\left(X_{(J)}^{T} X_{(J)}\right)^{-1}=\left(X^{T} X\right)^{-1}+\left(X^{T} X\right)^{-1} X_{J}^{T}\left(I_{k}-H_{J}\right)^{-1} X_{J}\left(X^{T} X\right)^{-1},
$$

where $H_{J}=X_{J}\left(X^{T} X\right)^{-1} X_{J}^{T}$. We write as $\hat{\beta}_{(J)}$ the least squares estimator of $\beta$ for the regression (2.1) computed without the cases indexed by $J$. Since $\left(I_{k}-H_{J}\right)^{-1}=I_{k}+\left(I_{k}-H_{J}\right)^{-1} H_{J}$ and $X_{(J)}^{T} y_{(J)}=$ $X^{T} y-X_{J}^{T} y_{J}$, a little computation yields

$$
\begin{aligned}
\hat{\beta}_{(J)} & =\left(X_{(J)}^{T} X_{(J)}\right)^{-1} X_{(J)}^{T} y_{(J)} \\
& =\hat{\beta}-\left(X^{T} X\right)^{-1} X_{J}^{T}\left(I_{k}-H_{J}\right)^{-1} e_{J},
\end{aligned}
$$

where $e_{J}=y_{J}-X_{J} \hat{\beta}$. Thus we have

$$
\hat{\beta}-\hat{\beta}_{(J)}=\left(X^{T} X\right)^{-1} X_{J}^{T}\left(I_{k}-H_{J}\right)^{-1} e_{J}
$$


The sampling distribution of $\hat{\beta}-\hat{\beta}_{(J)}$ is seen to be determined wholly by that of $e_{J}$ and we need to find it for our purpose. Since $e=\left(I_{n}-H\right) y$, the expectation of the residual vector $e$ is $E(e)=0$ and its covariance matrix is $\operatorname{cov}(e)=\sigma^{2}\left(I_{n}-H\right)$. We let $Q$ be a subsidiary matrix of size $k \times n$ that extracts the elements indexed by $J$ from the residual vector $e$. Since the rows of $Q$ are linearly independent, the rank of $Q$ is just $k$. We have $e_{J}=Q e$ which enables us to easily compute the covariance matrix of $e_{J}$ as

$$
\operatorname{cov}\left(e_{J}\right)=Q \operatorname{cov}(e) Q^{T}=\sigma^{2}\left(I_{k}-H_{J}\right)
$$

The expectation of $\hat{\beta}-\hat{\beta}_{(J)}$ is zero and its covariance matrix is computed as

$$
\operatorname{cov}\left(\hat{\beta}-\hat{\beta}_{(J)}\right)=\sigma^{2}\left(X^{T} X\right)^{-1} X_{J}^{T}\left(I_{k}-H_{J}\right)^{-1} X_{J}\left(X^{T} X\right)^{-1} .
$$

Since the rank of $C A C^{T}$ is equal to that of $C$ for a positive definite matrix $A$ and a matrix $C$ of an appropriate size, the rank of $\operatorname{cov}\left(\hat{\beta}-\hat{\beta}_{(J)}\right)$ is given by that of $\left(X^{T} X\right)^{-1} X_{J}^{T}$. Since $X^{T} X$ is nonsingular, the rank of $\left(X^{T} X\right)^{-1} X_{J}^{T}$ is equivalent to that of $X_{J}$ which is $\min \{k, p\}$. Hence the probability distribution of $\operatorname{cov}\left(\hat{\beta}-\hat{\beta}_{(J)}\right)$ resides wholly in a $\min \{k, p\}$-dimensional subspace of the $p$-dimensional Euclidean space.

The influence of deleting the observations corresponding to the index set $J$ on the least squares estimator $\hat{\beta}$ can be measured by the remoteness of $\hat{\beta}_{(J)}$ from $\hat{\beta}$. It is reflected in the covariance matrix $\operatorname{cov}\left(\hat{\beta}-\hat{\beta}_{(J)}\right)$, not in the mean $E\left(\hat{\beta}-\hat{\beta}_{(J)}\right)$ because the mean is always zero irrespective of deletions. Thus an influence analysis of deleting observations can be performed using the covariance matrix $\operatorname{cov}\left(\hat{\beta}-\hat{\beta}_{(J)}\right)$ to which the principal components analysis is applied in order to remove components along redundant axes if any, which will be described in what follows. Since the $\operatorname{rank}$ of $\operatorname{cov}(\hat{\beta}-$ $\left.\hat{\beta}_{(J)}\right)$ is $\min \{k, p\}\left(=m\right.$, say for convenience), it has $m$ positive eigenvalues $r_{1}, \ldots, r_{m}$, and the first $m$ standardized eigenvectors $g_{1}, \ldots, g_{m}$ associated with these eigenvalues describe the whole structure of the covariance matrix. The first $m$ principal components of $\hat{\beta}-\hat{\beta}_{(J)}$ are the associated coordinates with respect to the first $m$ eigenvectors $g_{1}, \ldots, g_{m}$, whose variances are the eigenvalues $r_{1}, \ldots, r_{m}$, respectively. The absolute values of the principal components of $\hat{\beta}-\hat{\beta}_{(J)}$ will become large as $\hat{\beta}_{(J)}$ stays away from $\hat{\beta}$, and so will their variances. Whenever $k<p$, there are $p-k$ redundant axes for describing the probability distribution of $\operatorname{cov}\left(\hat{\beta}-\hat{\beta}_{(J)}\right)$, and projections of $\hat{\beta}-\hat{\beta}_{(J)}$ along these redundant axes make no contribution to the influence of observations in $J$ on $\hat{\beta}$.

Since $\sigma^{2}$ appears commonly to all the case deletions, we can drop it for performing our principal components analysis based on $\operatorname{cov}\left(\hat{\beta}-\hat{\beta}_{(J)}\right)$. Let $\hat{r}_{1}, \ldots, \hat{r}_{m}$ be the $m$ positive eigenvalues of $\left(X^{T} X\right)^{-1} X_{J}^{T}\left(I_{k}-H_{J}\right)^{-1} X_{J}\left(X^{T} X\right)^{-1}$ and $\hat{g}_{1}, \ldots, \hat{g}_{m}$ be the associated standardized eigenvectors, respectively. We define

$$
\begin{aligned}
& R_{(J)}=\sum_{i=1}^{m} \hat{r}_{i}, \\
& V_{(J)}=\sum_{i=1}^{m}\left\{\hat{g}_{i}^{T}\left(\hat{\beta}-\hat{\beta}_{(J)}\right)\right\}^{2} .
\end{aligned}
$$

Since $R_{(J)}$ or $V_{(J)}$ or both will become large as $\hat{\beta}_{(J)}$ gets far from $\hat{\beta}$, a reasonable measure of the remoteness of $\hat{\beta}_{(J)}$ from $\hat{\beta}$ is to consider both $R_{(J)}$ and $V_{(J)}$. A graphical display of the pairs $\left(R_{(J)}, V_{(J)}\right)$ in the plane can be useful for identifying subsets of influential observations for each $k=1,2, \ldots$, in which subsets of observations located away from the origin are potentially influential. 


\section{Influence in testing}

\subsection{Test statistic}

We will review a procedure of checking whether an additional observation follows the regression model (2.1) and more details can be found in Chow (1960). Let $y_{n+1}$ be an observation on the response variable associated with the column vector $x_{n+1}$ of particular measurements on $p$ regressors. In order to check whether a particular single case $\left(y_{n+1}, x_{n+1}\right)$ comes from the regression (2.1), we will adopt the procedure described in what follows. First we assume that the case $\left(y_{n+1}, x_{n+1}\right)$ follows the regression model

$$
y_{n+1}=x_{n+1}^{T} \beta_{*}+\varepsilon_{n+1},
$$

where $\beta_{*}$ is a column vector of $p$ unknown regression coefficients and $\varepsilon_{n+1}$ is an unobservable random error distributed as $N\left(0, \sigma^{2}\right)$, independent of $\varepsilon$ in the regression (2.1). Then a hypothesis that a particular single case $\left(y_{n+1}, x_{n+1}\right)$ comes from the given regression (2.1) is equivalent to the following hypothesis

$$
H_{0}: \beta=\beta_{*} .
$$

A test of this hypothesis can be performed using the difference between $y_{n+1}$ and the predicted value at $x_{n+1}$ from the regression (2.1), and this difference can be written as

$$
\begin{aligned}
D & =y_{n+1}-x_{n+1}^{T} \hat{\beta} \\
& =x_{n+1}^{T}\left(\beta_{*}-\beta\right)+\epsilon_{n+1}-x_{n+1}^{T}\left(X^{T} X\right)^{-1} X^{T} \varepsilon .
\end{aligned}
$$

Hence we can easily see that the sampling distribution of the difference $D$ is a normal whose mean and variance are given by

$$
\begin{aligned}
E(D) & =x_{n+1}^{T}\left(\beta_{*}-\beta\right), \\
\operatorname{var}(D) & =\left[1+x_{n+1}^{T}\left(X^{T} X\right)^{-1} x_{n+1}\right] \sigma^{2},
\end{aligned}
$$

respectively. Under the null hypothesis $H_{0}$, the sampling distribution of the ratio

$$
T=\frac{n-p}{1+x_{n+1}^{T}\left(X^{T} X\right)^{-1} x_{n+1}} \frac{\left(y_{n+1}-x_{n+1}^{T} \hat{\beta}\right)^{2}}{s^{2}}
$$

is an $F$-distribution with degrees of freedom 1 and $n-p$. If the value of the test statistic $T$ is significantly large, we would reject the null hypothesis $H_{0}$.

\subsection{Multiple case deletions}

Using the identity in (3.1), we have

$$
x_{n+1}^{T}\left(X_{(J)}^{T} X_{(J)}\right)^{-1} x_{n+1}=x_{n+1}^{T}\left(X^{T} X\right)^{-1} x_{n+1}+x_{n+1}^{T}\left(X^{T} X\right)^{-1} X_{J}^{T}\left(I_{k}-H_{J}\right)^{-1} X_{J}\left(X^{T} X\right)^{-1} x_{n+1} .
$$


The residual sum of squares computed without the cases indexed by $J$ is computed as

$$
\begin{aligned}
s_{(J)}^{2} & =\left(y_{(J)}-X_{(J)} \hat{\beta}_{(J)}\right)^{T}\left(y_{(J)}-X_{(J)} \hat{\beta}_{(J)}\right) \\
& =y_{(J)}^{T} y_{(J)}-2 \hat{\beta}_{(J)}^{T} X_{(J)}^{T} y_{(J)}+\hat{\beta}_{(J)}^{T} X_{(J)}^{T} X_{(J)} \hat{\beta}_{(J)} \\
& =s^{2}-e_{J}^{T}\left[I_{k}+\left(I_{k}-H_{J}\right)^{-1} H_{J}\right] e_{J} \\
& =s^{2}-e_{J}^{T}\left(I_{k}-H_{J}\right)^{-1} e_{J} .
\end{aligned}
$$

The difference between $y_{n+1}$ and the predicted value at $x_{n+1}$ computed without the cases indexed by $I$ is given by

$$
\begin{aligned}
D_{(J)} & =y_{n+1}-x_{n+1}^{T} \hat{\beta}_{(J)} \\
& =D+x_{n+1}^{T}\left(X^{T} X\right)^{-1} X_{J}^{T}\left(I_{k}-H_{J}\right)^{-1} e_{J} .
\end{aligned}
$$

From (4.2) and (4.3), we get

$$
\begin{aligned}
& {\left[1+x_{n+1}^{T}\left(X_{(J)}^{T} X_{(J)}\right)^{-1} x_{n+1}\right] s_{(J)}^{2}} \\
& =\left[1+x_{n+1}^{T}\left(X^{T} X\right)^{-1} x_{n+1}\right] s^{2}-e_{J}^{T}\left(I_{k}-H_{J}\right)^{-1} e_{J} \\
& \quad+x_{n+1}^{T}\left(X^{T} X\right)^{-1} X_{J}^{T}\left(I_{k}-H_{J}\right)^{-1} X_{J}\left(X^{T} X\right)^{-1} x_{n+1}\left[s^{2}-e_{J}^{T}\left(I_{k}-H_{J}\right)^{-1} e_{J}\right] .
\end{aligned}
$$

When the cases indexed by $J$ are removed from the sample, the test statistic given in (4.1) is then computed as

$$
T_{(J)}=\frac{n-p-k}{1+x_{n+1}^{T}\left(X_{(J)}^{T} X_{(J)}\right)^{-1} x_{n+1}} \frac{D_{(J)}^{2}}{s_{(J)}^{2}}
$$

which can be evaluated using (4.4) and (4.5). The large absolute value of $T-T_{(J)}$ implies that the group effect of the cases indexed by $J$ on the test statistic $T$ can be high.

\section{A numerical example}

Single, double and triple case deletions are performed for the body fat data set (Neter et al., 1996, p.261) which have 20 measurements on a single dependent variable and three independent variables. It is assumed that the intercept term is included in the regression model. For our analysis, we divide the body fat data set into two groups: the first part consists of the first 19 observations and the second part comprises the last observation only. The null hypothesis $H_{0}$ is defined as one that the last observation follows the regression estimated by the first 19 observations, and we will investigate the influence of observation belonging to the first part in testing the null hypothesis $H_{0}$. Based on the first 19 observations, the least squares estimate of $\beta=\left(\beta_{0}, \beta_{1}, \beta_{2}, \beta_{3}\right)^{T}$ is computed as

$$
\beta_{0}=113.72, \quad \beta_{1}=4.23, \quad \beta_{2}=-2.77, \quad \beta_{3}=-2.13 .
$$

The value of the test statistic $T$ in (4.1) is computed as 0.061 and its associated $p$-value is 0.808 . Hence we can conclude at reasonable significance levels that the last observation follows the regression formed by the first 19 observations. 


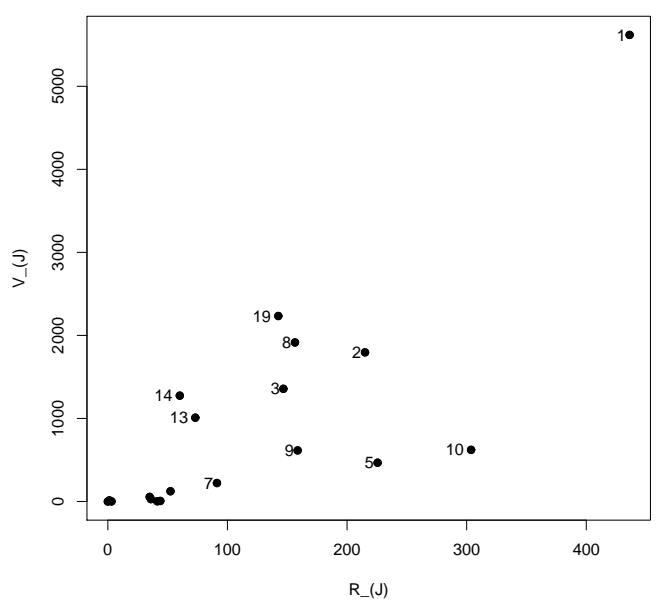

(a) Single case deletions

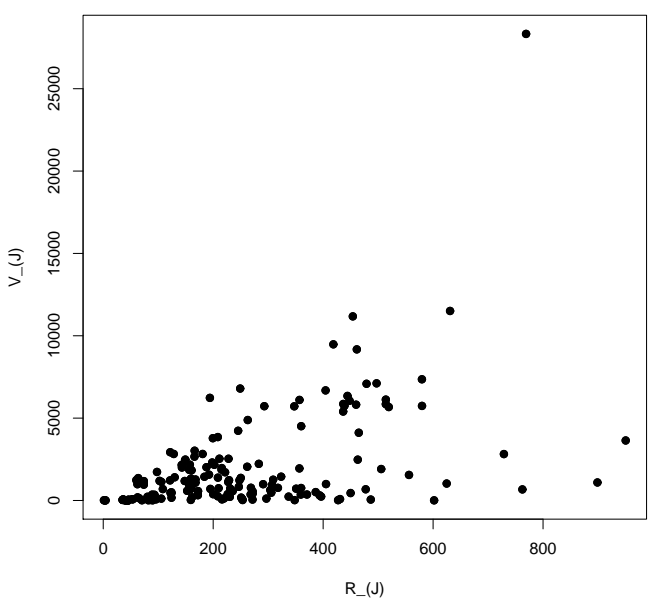

(b) Double case deletions

Figure 1: Graphical displays of the pairs $\left(R_{(J)}, V_{(J)}\right)$.

\subsection{Single case deletions}

For single case deletions, the corresponding graphical display of the pairs $\left(R_{(J)}, V_{(J)}\right)$ in the plane is included in Figure 1(a). Case 1 is remarkably distinct from the other cases. Cases 2, 8, 10, 19, etc are located far from the origin, lying on the outskirts of the figure. In order to confirm that the cases identified by Figure 1(a) are really influential ones, numerical single case deletions are performed and we investigated the absolute values of $\hat{\beta}_{i}-\hat{\beta}_{i(r)}(r=1,2, \ldots, 19)$ as follows.

1. For $\hat{\beta}_{0}$, case 1 is most influential and the next ones are in this order $19,8,2,3,14$, 13, etc. For each deletion of cases $1,19,8$ and $2, \hat{\beta}_{0}-\hat{\beta}_{0(r)}$ becomes $-74.9,-47.2,43.7$ and 42.3 , respectively.

2. For $\hat{\beta}_{1}$, case 1 is most influential and the next ones are in this order $19,8,2,14,13$, 3, etc. For each deletion of cases $1,19,8$ and $2, \hat{\beta}_{1}-\hat{\beta}_{1(r)}$ becomes $-2.17,-1.38,1.36$ and 1.27 , respectively.

3. For $\hat{\beta}_{2}$, case 1 is most influential and the next ones are in this order $19,8,2,3,14$, 13, etc. For each deletion of cases $1,19,8$ and $2, \hat{\beta}_{2}-\hat{\beta}_{2(r)}$ becomes $1.94,1.21,-1.16$ and -1.10 , respectively.

4. For $\hat{\beta}_{3}$, case 1 is most influential and the next ones are in this order $19,8,3,2,14,13$, etc. For each deletion of cases $1,19,3$ and $8, \hat{\beta}_{3}-\hat{\beta}_{3(r)}$ becomes $1.113,0.733,-0.678$ and -0.672 , respectively.

From this confirmation, we can see that Figure 1(a) provides quite accurate information about the influence of cases on $\hat{\beta}$.

Single case deletions of test statistic given in (4.6) are included in Table 1. The column with the heading $T$ shows the value of the test statistic computed without the corresponding case and the related $p$-value is in the column with the heading $p$. Table 1 shows that the removal of each of cases 19 and 1 increases the $p$-value compared with the others. On the other hand, the deletion of each of cases 8 and 2 causes a great decrease in the $p$-value compared with the others, and that of case 9 decreases the $p$-value in the third place. For single case deletions these influential cases in testing $H_{0}$ are also influential in estimating the regression coefficients. 
Table 1: Single case deletions of test statistic

\begin{tabular}{ccc|ccc|ccc}
\hline \hline No. & $T$ & $p$ & No. & $T$ & $p$ & No. & $T$ \\
\hline 1 & 0.005 & 0.947 & 8 & 0.151 & 0.703 & 15 & 0.069 & 0.797 \\
2 & 0.133 & 0.721 & 9 & 0.100 & 0.756 & 16 & 0.060 & 0.810 \\
3 & 0.048 & 0.830 & 10 & 0.058 & 0.814 & 17 & 0.055 & 0.818 \\
4 & 0.036 & 0.853 & 11 & 0.064 & 0.805 & 18 & 0.038 & 0.849 \\
5 & 0.063 & 0.806 & 12 & 0.088 & 0.772 & 19 & 0.012 & 0.915 \\
6 & 0.051 & 0.824 & 13 & 0.045 & 0.835 & & & \\
7 & 0.066 & 0.800 & 14 & 0.110 & 0.745 & & & \\
\hline \hline
\end{tabular}

Table 2: $\left(R_{(J)}, V_{(J)}\right)$ for some double case deletions

\begin{tabular}{c|cccc}
\hline \hline Cases & $(1,19)$ & $(1,10)$ & $(1,2)$ & $(1,8)$ \\
\hline$\left(R_{(J)}, V_{(J)}\right)$ & $(769.1,28326.9)$ & $(950.3,3643.0)$ & $(898.9,1093.5)$ & $(762.4,671.9)$ \\
\hline Cases & $(1,9)$ & $(1,7)$ & $(2,8)$ & $(5,14)$ \\
\hline$\left(R_{(J)}, V_{(J)}\right)$ & $(728.8,2818.1)$ & $(630.8872,11506.5)$ & $(454.0,11176.8)$ & $(418.6,9479.7)$ \\
\hline \hline
\end{tabular}

Table 3: Double case deletions of test statistic

\begin{tabular}{ccc|ccc}
\hline \hline No. & $T$ & $p$ & No. & $T$ & $p$ \\
\hline$(1,4)$ & 0.000 & 0.997 & $(2,8)$ & 0.343 & 0.568 \\
$(1,19)$ & 0.055 & 0.817 & $(4,19)$ & 0.002 & 0.970 \\
\hline \hline
\end{tabular}

\subsection{Double case deletions}

For double case deletions, Figure 1(b) includes the corresponding graphical display of the pairs $\left(R_{(J)}, V_{(J)}\right)$ in the plane. Some double case deletions lying on the outskirts of Figure 1(b) that are far from the origin are summarized in Table 2. Deletion of double cases $(1,19)$ is remarkably distinct from the others. We perform numerical double case deletions to show the efficiency of Figure 1(b) based on the absolute value of $\hat{\beta}_{i}-\hat{\beta}_{i(J)}$ and they are summarized as follows.

1. For $\hat{\beta}_{0}$, deletions of double cases $(1,19),(1,7),(2,8)$ are highly influential in this order. For each deletion of double cases $(1,19),(1,7)$ and $(2,8), \hat{\beta}_{0}-\hat{\beta}_{0(J)}$ becomes $-168.2,-107.2$ and 105.6, respectively.

2. For $\hat{\beta}_{1}$, deletions of double cases $(1,19),(2,8),(5,14),(1,7)$ are highly influential in this order. For each deletion of double cases $(1,19),(2,8),(5,14)$ and $(1,7), \hat{\beta}_{1}-\hat{\beta}_{1(J)}$ becomes $-4.90,3.23$, -3.14 and -3.10 , respectively.

3. For $\hat{\beta}_{2}$, deletions of double cases $(1,19),(2,8),(1,7),(5,14)$ are highly influential in this order. For each deletion of double cases $(1,19),(2,8),(1,7)$ and $(5,14), \hat{\beta}_{2}-\hat{\beta}_{2(J)}$ becomes $4.33,-2.77$, 2.77 and 2.52 , respectively.

4. For $\hat{\beta}_{3}$, deletions of double cases $(1,19),(5,14),(2,8),(1,7),(1,14)$ are highly influential in this order. For each deletion of double cases $(1,19),(5,14),(2,8),(1,7)$ and $(1,14), \hat{\beta}_{3}-\hat{\beta}_{3(J)}$ becomes $2.54,1.75,-1.64,1.59$ and 1.53 , respectively.

Some double case deletions which yield significant changes in the value of test statistic are included in Table 3. The $p$-values over all double case deletions range from 0.568 to 0.997. Each removal of double cases $(1,4)$ or $(4,19)$ does not allow us to reject the null hypothesis at any reasonable significance levels, and that of double cases $(2,8)$ decreases the $p$-value. Though each single 
Table 4: $\left(R_{(J)}, V_{(J)}\right)$ for some triple case deletions

\begin{tabular}{c|cccc}
\hline \hline Cases & $(1,7,19)$ & $(1,2,10)$ & $(1,2,8)$ & $(1,2,19)$ \\
\hline$\left(R_{(J)}, V_{(J)}\right)$ & $(1026,46005)$ & $(1631,31.1)$ & $(1515,2550)$ & $(1523,23152)$ \\
\hline Cases & $(1,8,10)$ & $(1,2,9)$ & $(1,10,19)$ & $(1,9,19)$ \\
\hline$\left(R_{(J)}, V_{(J)}\right)$ & $(1448,13.4)$ & $(1414,52.4)$ & $(1416,28876)$ & $(1248,27560)$ \\
\hline \hline
\end{tabular}

deletion of cases 1 and 19 greatly increases the $p$-value, the removal of double cases $(1,19)$ does not cause a noticeable change in the $p$-value, that is the joint influence of cases 1 and 19 on the test statistic is not severe.

\subsection{Triple case deletions}

For triple case deletions, a graphical display of the pairs $\left(R_{(J)}, V_{(J)}\right)$ in the plane, nor provided here, shows that the deletion of triple cases $(1,7,19)$ is separated from the main body of the remaining data. Some observations residing in the outskirts of the main body are listed in Table 4.

1. For $\hat{\beta}_{0}$, the values of $\hat{\beta}_{0}-\hat{\beta}_{0(J)}$ over all triple case deletions range from -214.3 to 157.3 . The first three most influential subsets are as follows: $\hat{\beta}_{0}-\hat{\beta}_{0(J)}$ for each deletion of $(1,7,19),(1,18,19)$ and $(1,14,19)$ becomes $-214.3,-181.7$ and -180.7 , respectively.

2. For $\hat{\beta}_{1}$, the values of $\hat{\beta}_{1}-\hat{\beta}_{1(J)}$ over all triple case deletions range from -6.22 to 4.73 . The first three most influential subsets are as follows: $\hat{\beta}_{1}-\hat{\beta}_{1(J)}$ for each deletion of $(1,7,19),(1,14,19)$ and $(1,18,19)$ becomes $-6.22,-5.38$ and -5.33 , respectively.

3. For $\hat{\beta}_{2}$, the values of $\hat{\beta}_{2}-\hat{\beta}_{2(J)}$ over all triple case deletions range from -4.067 to 5.513 . The first three most influential subsets are as follows: $\hat{\beta}_{2}-\hat{\beta}_{2(J)}$ for each deletion of $(1,7,19),(1,18,19)$ and $(1,14,19)$ becomes $5.513,4.664$ and 4.662 , respectively.

4. For $\hat{\beta}_{3}$, the values of $\hat{\beta}_{3}-\hat{\beta}_{3(J)}$ over all triple case deletions range from -2.48 to 3.22 . The first three most influential subsets are as follows: $\hat{\beta}_{0}-\hat{\beta}_{0(J)}$ for each deletion of $(1,7,19),(1,14,19)$ and $(1,18,19)$ becomes $3.22,2.82$ and 2.80 , respectively.

Thus we can see that the deletion of triple cases $(1,7,19)$ is most influential on all of the regression coefficients.

The $p$-values over all triple case deletions range from 0.45983 to 0.99993 . The deletion of triple cases $(1,6,18)$ increases the $p$-value from 0.808 to 0.99993 while the removal of triple cases $(2,8,9)$ decreases the $p$-value from 0.808 to 0.45983 . The dramatic change from the acceptance of $H_{0}$ to its rejection can not occur due to any triple case deletion. The deletion of triple cases $(1,7,19)$ decreases the $p$-value from 0.808 to 0.797 , but this change is negligible. Hence the deletion of triple cases $(1,7$, 19 ) is influential in estimating all of the regression coefficients but not in testing $H_{0}$.

\section{Concluding remarks}

We note that no assumption about a distributional form is in fact needed just for applying the diagnostic method introduced in Section 3. The diagnostic statistic $\hat{\beta}-\hat{\beta}_{(J)}$ is a vector. It is usually normalized so that subsets of observations can be ordered in a meaningful way (Chatterjee and Hadi, 1988). For example one popular normalized diagnostic statistic is the Cook's distance (Cook, 1977). However, the use of the Cook's distance sometimes fails to identify influential observations correctly 
as Kim (2015) has shown, where some results relevant to a single case deletion can also be found. Our diagnostic method does not need any normalization.

The diagnostic method introduced in Section 3 can be used for any statistical problems where the covariance matrix of $\hat{\beta}-\hat{\beta}_{(J)}$ is available theoretically or numerically.

\section{References}

Chatterjee S and Hadi AS (1988). Sensitivity Analysis in Linear Regression, John Wiley \& Sons, New York.

Chow GC (1960). Tests of equality between sets of coefficients in two linear regressions, Econometrica, 28, 591-605.

Cook RD (1977). Detection of influential observation in linear regression, Technometrics, 19, 15-18.

Cook RD and Weisberg S (1982). Residuals and Influence in Regression, Chapman and Hall, New York.

Ghilagaber G (2004). Another look at Chow's test for the equality of two heteroscedastic regression models, Quality and Quantity, 38, 81-93.

Kim MG (2015). Influence measure based on probabilistic behavior of regression estimators, Computational Statistics, 30, 97-105.

Neter J, Kutner MH, Nachtsheim CJ, and Wasserman W (1996). Applied Linear Regression Models (3rd ed), McGraw-Hill Higher Education/Irwin, New York.

Seber GAF (1977). Linear Regression Analysis, John Wiley \& Sons, New York.

Received March 15, 2016; Revised April 12, 2016; Accepted April 14, 2016 